\title{
From the Circumnuclear Disk in the Galactic Center to thick, obscuring tori of AGNs
}

\author{
B. Vollmer ${ }^{1,2}$, T. Beckert ${ }^{2}$, and W. J. Duschl $1^{3,2}$ \\ ${ }^{1}$ CDS, Observatoire astronomique de Strasbourg, UMR 7550, 11 rue de l'Université, 67000 Strasbourg, France \\ 2 Max-Planck-Institut für Radioastronomie, Auf dem Hügel 69, 53121 Bonn, Germany \\ 3 Institut für Theoretische Astrophysik der Universität Heidelberg, Tiergartenstraße 15, 69121 Heidelberg, Germany
}

Received 15 August 2003 / Accepted 6 October 2003

\begin{abstract}
We compare three different models of clumpy gas disk and show that the Circumnuclear Disk (CND) in the Galactic Center and a putative, geometrically thick, obscuring torus are best explained by a collisional model consisting of quasi-stable, self-gravitating clouds. Kinetic energy of clouds is gained by mass inflow and dissipated in cloud collisions. The collisions give rise to a viscosity in a spatially averaged gas dynamical picture, which connects them to angular momentum transport and mass inflow. It is found that CND and torus share the same gas physics in our description, where the mass of clouds is $20-50 M_{\odot}$ and their density is close to the limit of disruption by tidal shear. We show that the difference between a transparent CND and an obscuring torus is the gas mass and the velocity dispersion of the clouds. A change in gas supply and the dissipation of kinetic energy can turn a torus into a CND-like structure and vice versa. Any massive torus will naturally lead to sufficiently high mass accretion rates to feed a luminous AGN. For a geometrically thick torus to obscure the view to the center even super-Eddington accretions rates with respect to the central black hole are required.
\end{abstract}

Key words. ISM: clouds - ISM: structure - Galaxy: structure - Galaxy: center - galaxies: nuclei

\section{Introduction}

The central black hole of the Galaxy is surrounded by several hundred clouds of gas and dust forming a disk-like structure (Circumnuclear Disk CND, see e.g. Güsten et al. 1987) up to a radius of $\sim 7 \mathrm{pc}^{1}$. The CND has a hydrogen mass of a few times $10^{4} M_{\odot}$ which is distributed in clumps with an estimated area filling factor of $\phi_{\mathrm{A}} \sim 0.1$ and a volume filling factor of $\phi_{\mathrm{V}} \sim 0.01$. The clumps have densities of several $10^{5} \mathrm{~cm}^{-3}$, radii of $\sim 0.05 \mathrm{pc}$ and gas temperatures $\geq 100 \mathrm{~K}$. A typical dusty clump has a visual extinction $A_{\mathrm{V}}>30^{\mathrm{m}}$ and $M_{\mathrm{H}} \sim 30 M_{\odot}$. Further properties of the clouds in the central $2 \mathrm{pc}$ are listed in Jackson et al. (1993). The vertical thickness $(2 H)$ of the CND increases from $\sim 0.5 \mathrm{pc}$ at a radius of $2 \mathrm{pc}$ to about $2 \mathrm{pc}$ at the outer radius of $7 \mathrm{pc}$. The disk rotates with a velocity of $\sim 100 \mathrm{~km} \mathrm{~s}^{-1}$ which corresponds to a Keplerian velocity around a central object of several $10^{6} M_{\odot}$ and has a velocity dispersion of $\sim 20 \mathrm{~km} \mathrm{~s}^{-1}$. With an $R / H$ of $\sim 5-7$ ( $R$ being the radius to the center), the CND can be qualified as a thin disk. At a distance of $\sim 1.5 \mathrm{pc}$ the molecular gas density drops by more than a magnitude, i.e. the central $3 \mathrm{pc}$ of the Galaxy are

Send offprint requests to: T. Beckert,

e-mail: tbeckert@mpifr-bonn.mpg.de

1 We assume $8.5 \mathrm{kpc}$ for the distance to the Galactic Center. void of molecular gas. In this sense the CND resembles more a ring or a torus.

In active galactic nuclei (AGN) the central luminous and compact object (e.g. the inner accretion disk around the black hole) is likely to be obscured by the torus, if the mean free path of clouds along the line of sight is greater than one. This qualitatively obvious statement was quantified by Nenkova et al. (2002) based on the comparison between models of cloudy dust emission and IR spectral energy distributions. They conclude that the number of clouds along the line of sight should be 5-10, which corresponds to the mean free path of clouds being 5-10 times smaller than the radius of the torus. Krolik \& Begelman (1988) were among the first to discuss the overall physical properties of the circumnuclear gas in active galactic centers. They already emphasized the importance of the cloudy structure of the circumnuclear material.

Based on the unified model (Antonucci 1993) the spectroscopic difference between Seyfert 1 and 2 nuclei is due to the line of sight passing through the torus (Sy 2) or not (Sy 1). From the relative numbers of Sy 1 and Sy 2, Schmitt et al. (2001) derive a half-opening angle of $48^{\circ}$ for the obscuring torus. But Tran (2003) shows in a recent paper that about half the Sy 2 nuclei derived from a $12 \mu \mathrm{m}$ sample do not have detectable hidden broad line regions (HBLR) in spectropolarimetric observations. Because the obscuration determined 
from X-ray spectra is not systematically different for Sy 2 with HBLR and those without HBLR, it is not obvious that the obscuring material in non-HBLR Sy2 nuclei, which seem to be intrinsically fainter, is in form of a torus. It is therefore difficult to determine the half-opening angle of the torus and its relative thickness statistically.

More evidence can be found by imaging extended NLR in OIII for example. For some sources the extended NLR take the shape of an ionization cone either due to a anisotropic radiation source or a collimating torus (for a review see Wilson 1996; Mulchaey et al. 1996 for a list of morphologically identified sources). The latter scenario is supported by evidence for obscuration at the apex of some of the observed cones. If this torus is responsible for an ionization cone, it extends radially from a few pc to several tens of pc and its flaring should be $\alpha=\left(180^{\circ}-\psi\right) / 2$, where $\psi$ is the opening angle of the ionization cone. With a mean opening angle of $\bar{\psi}=80^{\circ} \pm 20^{\circ}$, the disk flaring angle is $\alpha \sim 50^{\circ} \pm 10^{\circ}$ or $H / R \sim 1$, which means that these tori are geometrically thick.

The CND in the Galactic Center in its current form is clearly not an obscuring torus, because the mean free path of the clouds along the radius is $l_{\mathrm{cl}} \sim 10 \mathrm{pc}$ and thus $R / l_{\mathrm{cl}}<$ 1 , where $R=7 \mathrm{pc}$ is the extent of the CND, i.e. the line of sight through the torus. It is not a thick disk either, because $H / R \sim 0.2$.

In this article we investigate if there are common properties between the CND in the Galactic Center and obscuring tori in Seyfert galaxies. In Sect. 2 we recall the theory of clumpy accretion disks. The equations needed for the comparison between the different models and observations are given in Sect. 3. The models are then applied to the CND and a thick, obscuring torus (Sect. 4). We discuss the results in Sect. 5 and give our conclusions in Sect. 6.

\section{The theory of clumpy gas disks}

\subsection{Turbulent disks}

In a recent work (Vollmer \& Beckert 2002, Paper I) we developed an analytical model for clumpy accretion disks and included a simplified description of turbulence in the disk. In contrast to classical accretion disk theory (see, e.g., Pringle 1981), we do not use the "thermostat" mechanism, which implies a direct coupling between the heat produced by viscous friction and the viscosity itself which is assumed to be proportional to the thermal sound speed. Thus, the viscosity, which is responsible for the gas heating, depends itself on the gas temperature. This leads to an equilibrium corresponding to a thermostat mechanism. Instead, we used an energy flux conservation, where the potential energy that is gained through mass accretion and differential rotation is cascaded by turbulence from large to small spatial scales. The inertial range of the turbulent cascade under consideration will not reach down to the scale of microscopic dissipation. Instead we regard the energy to be dissipated at an inner scale where the first cascade coming from the largest scales ends or breaks.

In a first approximation this happens when the gas clouds become self-gravitating and dissipation then takes place inside individual clouds. Because the energy reservoir is the gravitational binding energy in the potential of the galaxy, a star cluster, or a central black hole, and the dissipation scale is set by self-gravity, we will call this scenario the fully gravitational model (FG model) in the following. Turbulence transfers the energy from the driving wavelength $l_{\text {driv }}$ to the dissipation wavelength $l_{\text {diss }}$, which corresponds to the length scale of a self-gravitating cloud. The two length scales are linked by the scaling parameter $\zeta$ which measures the extent of the inertial range. For a Kolmogorov-like turbulent energy spectrum $\zeta=\left(l_{\text {driv }} / l_{\text {diss }}\right)^{\frac{3}{4}}$.

An additional "free" parameter of the model is the Toomre- $Q$ parameter:

$Q=\frac{v_{\text {turb }} \Omega}{\pi G \Sigma} \geq 1$

where $v_{\text {turb }}$ is the turbulent velocity, $\Omega$ the angular velocity, $G$ the gravitational constant, and $\Sigma$ the gas surface density of the disk. For all models we assume $Q$ to be constant throughout the disk. If we can approximate the total gas mass within a radius $R$ by $M_{\text {gas }}=\pi R^{2} \Sigma$ Toomre- $Q$ can be rewritten

$Q=\frac{v_{\text {turb }}}{v_{\text {rot }}} \frac{M_{\mathrm{tot}}}{M_{\mathrm{gas}}}$

where $M_{\mathrm{tot}}$ is the total enclosed mass and $v_{\text {rot }}$ the rotation velocity.

Like in a standard accretion disk scenario a viscosity allows redistribution of angular momentum and mass accretion together with a gain of kinetic energy from the potential well in which the disk resides. For the viscosity we use the following prescription:

$v=\zeta^{-1} v_{\text {turb }} l_{\text {driv }}$.

A set of equations is obtained with 3 parameters: the Toomre parameter $Q$, the scaling parameter $\zeta$, and the mass accretion rate within the disk $\dot{M}$. These equations can be solved analytically and the results were already used in Paper I to describe properties of our Galaxy's extended gas disk. Besides the parameters above the solutions depend on the $v_{\text {rot }}$, and $R$. Within the model it turned out that the driving wavelength in Eq. (3) equals the disk height $l_{\text {driv }}=H$.

In a second step we included the energy input due to supernova (SN) explosions (Vollmer \& Beckert 2003, Paper II). Here the energy flux provided by $\mathrm{SNe}$ is transfered by turbulence to smaller scales where it is again dissipated. The SN energy flux is assumed to be proportional to the local star formation rate $\dot{\rho}_{*}$, which, in turn, is taken to be proportional to the mean density and inversely proportional to the local free fall time of clouds. These clouds have sizes that are a factor $\delta$ smaller than the driving length scale. The factor of proportionality is the probability to find a self-gravitating cloud, i.e. the volume filling factor. Because the model works with vertically integrated quantities, like the surface density, the integration length in the vertical direction is assumed to be the turbulent driving length scale, i.e. the vertical length scale over which clouds can become selfgravitating: $\dot{\Sigma}_{*}=\dot{\rho}_{*} l_{\text {driv }}$. The SN energy per unit time $\dot{E}_{\mathrm{SN}}$ per 
area $\Delta A$ is therefore proportional to the local star formation rate $\dot{\Sigma}_{*}$ :

$$
\frac{\dot{E}_{\mathrm{SN}}}{\Delta A}=\xi \dot{\Sigma}_{*},
$$

where the factor of proportionality $\xi$ is independent of the radius in the disk. Its normalization with Galactic values yields $\xi=4.6 \times 10^{-8}(\mathrm{pc} / \mathrm{yr})^{2}$.

In the FG model it is assumed that the energy flux transported through the turbulent cascade is supplied by mass accretion and the energy balance is

$\rho v \frac{v_{\text {turb }}^{2}}{l_{\text {driv }}}=-\frac{1}{2 \pi} \dot{M} v_{\text {rot }} \frac{\partial \Omega}{\partial R}$.

In the case of SN driven turbulence the energy flux is determined by the star formation rate

$\rho v \frac{v_{\text {turb }}^{2}}{l_{\text {driv }}}=\xi \dot{\Sigma}_{*}$.

In most gas disks on galactic scales the energy supply by supernovae dominates the gravitationally released energy for the same disk properties except for large $Q$ values. The situation changes if the disks are so thin, that SN bubbles break out and most of their energy is lost from the disk.

Furthermore, we take into account that the clouds are formed at the compressed edges during the interaction between $\mathrm{SN}$ remnants. The size of the clouds $l_{\mathrm{cl}}$ is smaller than the turbulent driving wavelength and we use $\delta=l_{\text {driv }} / l_{\mathrm{cl}}$ with $\delta \geq 1$.

\subsection{Collisional disks}

While the turbulent disk model describes the ISM as a continuous medium where a turbulent cascade can develop, this changes when the self-gravitating clouds become stable. In such a medium an equilibrium disk can form, if there are fragmenting or partially elastic collisions (the clouds are magnetized) between the clouds. These collisions give rise to a viscosity which depends on the velocity dispersion of the clouds (we will call it $v_{\text {turb }}$ nonetheless) and the mean free path of cloud with respect to cloud collisions $l_{\text {coll }}$. If the collisional timescale $t_{\text {coll }}=l_{\text {coll }} / v_{\text {turb }}$ is larger or equal to the dynamical timescale, the resulting viscosity (Pringle 1981) can be written as

$v=\tau^{-1} v_{\text {turb }} H$,

where $\tau=t_{\text {coll }} \Omega$ and $H$ is the disk height. Here it is already assumed that the disk is in hydrostatic equilibrium vertically $H=v_{\text {turb }} / \Omega$, where the pressure is provided by the velocity dispersion. The energy dissipation rate due to collisions can be written as

$$
\frac{\Delta E}{\Delta A \Delta t}=\epsilon \frac{\frac{3}{2} \Sigma v_{\text {turb }}^{2}}{t_{\text {coll }}}=\epsilon \frac{\frac{3}{2} \Sigma v_{\text {turb }}^{3}}{l_{\text {coll }}}=\Sigma v\left(R \frac{\partial \Omega}{\partial R}\right)^{2}
$$

The last equation on the right implies that the energy source is differential rotation and accretion as in the FG model. Here we introduced an efficiency parameter $\epsilon$ which measures the fraction of the kinetic energy of clouds dissipated in a typical collision. Because most conclusions from this model are independent of the particular form of the energy Eq. (8) we set $\epsilon=2 / 3$ and so we are consistent with Eq. (5) and the use in Paper I. Since the driving wavelength in Eq. (3) for the FG model is the disk scale height $l_{\text {driv }}=H$, the FG model and the collisional model are formally equivalent when we identify $\tau$ with $\zeta$. But the interpretation of the viscosity in terms of a continuous turbulent medium (FG model) and the collisional viscosity here are completely different.

\section{A comparison of characteristic properties of the three models}

In this section we recall relevant relations between observables within the FG and the SN models and derive observable properties of the collisional disk. This will allow us later on to determine the free model parameters and compare the models with observations.

\subsection{The fully gravitational (FG) model}

Following Paper I this model has two dimensionless parameters: Toomre- $Q$ defined in Eq. (2) and $\zeta$ in Eq. (3). From a solution consistent with observations it is possible to derive the mass accretion rate in the disk $\dot{M}$ from the FG model. Very importantly the turbulent velocity dispersion can be expressed as a function of the gravitational constant $G$, the scaling parameter $\zeta$, the mass accretion rate $\dot{M}$, and $Q$ :

$v_{\text {turb }}=\left(\frac{1}{2} G \zeta Q \dot{M}\right)^{\frac{1}{3}}$.

Furthermore the cloud radius is given by

$r_{\mathrm{cl}}=\zeta^{-1} H$,

and the volume filling factor, which is defined by $\phi_{\mathrm{V}}=\rho / \rho_{\mathrm{cl}}$, where $\rho$ is the mean density in the disk and $\rho_{\mathrm{cl}}$ is the density of the clouds, yields:

$\phi_{\mathrm{V}}=\frac{1}{\zeta Q}$.

For a disk with almost constant surface density the total gas mass is given by

$M_{\text {gas }}=\pi \Sigma R^{2}=2^{-\frac{1}{3}} G^{-\frac{2}{3}} \zeta^{\frac{1}{3}} Q^{-\frac{2}{3}} \dot{M}^{\frac{1}{3}} v_{\text {rot }} R$

where the rotational velocity $v_{\text {rot }}$ can be either directly observed or determined from the total mass distribution. In the case of an isothermal star cluster the surface density also has a cusp with $\Sigma \propto R^{-1}$ and the enclosed gas mass is a factor 2 larger than in Eq. (12).

\subsection{The SN model}

In Paper II we distinguish two cases: (i) a self-gravitating gas disk in vertical direction $(Q=1)$ and (ii) $Q>1$.

For $Q=1$ the velocity dispersion in the disk is given by

$v_{\text {turb }}=0.82 G^{\frac{3}{\pi}} \dot{M}^{\frac{3}{1}} \delta^{-\frac{1}{\pi}} \xi^{\frac{1}{\pi}}$,

where $\xi$ is the normalization of the SN energy input in Eq. (6) and $\delta$ the ratio if actual cloud size to the driving length scale. 
For $Q>1$ the velocity dispersion in the disk and the driving length scale are

$v_{\text {turb }}=0.87 \chi^{\frac{4}{15}} G^{\frac{1}{5}} \dot{M}^{\frac{1}{5}} Q^{\frac{4}{15}} \delta^{-\frac{1}{15}} \xi^{\frac{1}{15}} v_{\text {rot }}^{\frac{4}{15}}$,
$l_{\text {driv }}=0.69 \chi^{-\frac{1}{6}} G^{\frac{1}{2}} \dot{M}^{\frac{1}{2}} Q^{\frac{5}{6}} \delta^{\frac{1}{6}} \xi^{-\frac{1}{6}} v_{\text {rot }}^{-\frac{7}{6}} R$,

where $\chi=H / R$ is the relative thickness of the disk. The volume filling factor turns out to be

$\phi_{\mathrm{V}}=0.79 \chi^{-\frac{2}{15}} G^{\frac{2}{5}} \dot{M}^{\frac{2}{5}} Q^{-\frac{2}{15}} \delta^{-\frac{22}{15}} \xi^{-\frac{8}{15}} v_{\mathrm{rot}}^{-\frac{2}{15}}$.

It is possible to give a criterion for the validity of the model that is when the energy input through supernovae explosions Eq. (6) dominates over the gravitationally released energy due to accretion (Eq. (5)). This can be turned into the following condition for the volume filling factor:

$\phi_{\mathrm{V}}>\xi^{-2} \Omega^{-4} v_{\text {turb }}^{8} l_{\text {driv }}^{-4}$

\subsection{The collisional model}

For the collisional model we can get quite general relations for cloud sizes and a criterion for the $Q$ parameter to be satisfied so that clouds are actually stable. As already mentioned in Sect. 2.2, the mean free path of a cloud is

$l_{\text {coll }}=v_{\text {turb }} t_{\text {coll }}=\tau \frac{v_{\text {turb }}}{\Omega}=\tau H$,

while the mean free path on the other hand is the inverse of the cloud number density $n$ times the cloud cross section $\sigma=\pi r_{\mathrm{cl}}^{2}$ :

$l_{\mathrm{coll}}=(n \sigma)^{-1}=\left(\frac{3 \Sigma \phi_{\mathrm{V}}}{4 r_{\mathrm{cl}} \rho H}\right)^{-1}=\frac{4 r_{\mathrm{cl}}}{3 \phi_{\mathrm{V}}}$.

In contrast to the turbulent models, the condition for clouds to be self-gravitating is that the free fall time equals the sound crossing time (in the case of the turbulent models it is the turbulent crossing time):

$t_{\mathrm{ff}}=\sqrt{\frac{3 \pi}{32 G \rho_{\mathrm{cl}}}}=t_{\mathrm{sound}}=\frac{r_{\mathrm{cl}}}{c_{\mathrm{s}}}$,

where $c_{\mathrm{s}}$ is the sound speed within the clouds. Inserting $\rho=$ $\Omega^{2} /(\pi G Q)$ from the definition of the $Q$-parameter (Paper I) leads to

$r_{\mathrm{cl}}=\frac{\pi^{2}}{8} \frac{Q}{\tau} \Omega^{-1} v_{\mathrm{turb}}^{-1} c_{\mathrm{s}}^{2}$

Not surprisingly the size of clouds gets larger when the disk is further away from the gravitational instability (larger $Q$ values) and the collision time is long. The critical density against tidal shear is $\rho_{\text {crit }}=3 \Omega^{2} /(4 \pi G)$ and thus $\rho / \rho_{\text {crit }}=4 /(3 Q)$. On the other hand we have $\rho / \rho_{\mathrm{cl}}=\phi_{\mathrm{V}}$. The condition for the clouds to resist disruption by tidal shear is $\rho_{\mathrm{cl}}>\rho_{\text {crit }}$, which leads with the help of Eq. (20) to an upper bound for the cloud size:

$r_{\mathrm{cl}}<\frac{\pi}{\sqrt{8}} \frac{c_{\mathrm{s}}}{\Omega}$
Table 1. Observed properties for the CND.

\begin{tabular}{lll}
\hline \hline cloud radius & $r_{\mathrm{cl}}$ & $0.05 \mathrm{pc}$ \\
cloud mass & $M_{\mathrm{cl}}$ & $30 M_{\odot}$ \\
sound speed within the clouds & $c_{\mathrm{s}}$ & $1.05 \mathrm{~km} \mathrm{~s}^{-1}$ \\
turbulent velocity dispersion in the disk & $v_{\mathrm{turb}}$ & $20 \mathrm{~km} \mathrm{~s}^{-1}$ \\
disk radius & $R$ & $7 \mathrm{pc}$ \\
disk height & $H$ & $1 \mathrm{pc}$ \\
disk mass & $M_{\mathrm{gas}}$ & $2 \times 10^{4} M_{\odot}$ \\
rotation velocity & $v_{\mathrm{rot}}$ & $115 \mathrm{~km} \mathrm{~s}^{-1}$ \\
\hline
\end{tabular}

Inserting Eq. (21) for the cloud size gives the following condition for the ratio of Toomre parameter to the dimensionless collision time $\tau$

$\frac{Q}{\tau}<\frac{\sqrt{8}}{\pi} \frac{v_{\text {turb }}}{c_{\mathrm{s}}}$

or using the definition of the Toomre- $Q$ (Eq. (1)) a lower bound on the collision time for stability of clouds

$\tau>\frac{\Omega c_{\mathrm{s}}}{\sqrt{8} G \Sigma}$.

The upper bound on $Q / \tau$ increases for thick disks (e.g. large velocity dispersions), while the limit on the collision time gets shorter for more massive disks.

The limit on $Q / \tau$ is especially interesting for geometrically thick and obscuring tori, because obscuration requires $\tau=l_{\text {coll }} / H$ to be small, which turns into a severe constrain on the allowed $Q$-values.

For the CND the clumpy structure is allowed to be transparent, but with the known cloud sizes and temperatures (sound speed) it is possible to get a measure of $\tau$ from the formally equivalent FG model expressions Eqs. (9) and (12) by replacing $\zeta$ by $\tau$, and Eq. (21)

$\tau=\frac{\pi^{2}}{8} \frac{c_{\mathrm{s}}^{2}}{G M_{\mathrm{gas}}} \frac{R^{2}}{r_{\mathrm{cl}}}$

Obviously the collision time gets longer for smaller clouds, less massive disk, but surprisingly also for higher temperatures in the clouds.

\section{Finding the most appropriate model}

In this section we will determine the model parameters of the 3 different models for (i) the Circumnuclear Disk in the Galactic Center and (ii) for obscuring tori. This will enable us to discuss, which models are realistic for the two kinds of ring structures.

\subsection{What it needs to make a Circumnuclear Disk}

The observed or from observations deduced properties of the CND are summarized in Table 1 . The CND resides in a region where the central star cluster starts to dominate the total mass (e.g. Schödel et al. 2002). The total enclosed mass at radius $R$ 
rises linearly with $R$ and the rotation velocity is almost constant throughout the CND. Because the CND extends only over a limited range in radius we use $M_{\text {gas }}=\pi \Sigma R^{2}$ for the gas mass contrary to the discussion at the end of Sect. 3.1. For all models the Toomre- $Q$ parameter is

$Q=\frac{v_{\text {turb }} v_{\text {rot }} R}{G M_{\text {gas }}}=190$.

With this number fixed we can go to the individual models

\subsubsection{Massive clouds in the FG model}

From the cloud size and the disk height inserted in Eq. (10) we get $\zeta=20$. Together with the $Q$-value this leads to a volume filling factor $\phi_{\mathrm{V}}=(\zeta Q)^{-1} \approx 2.6 \times 10^{-4}$. Independent of the volume filling factor we find from Eq. (9) a mass accretion rate of $\dot{M}=10^{-3} M_{\odot} \mathrm{yr}^{-1}$. The mass of a single cloud follows $M_{\mathrm{cl}}=(4 / 3) \pi r_{\mathrm{cl}}^{3} \rho / \phi_{\mathrm{V}}=260 M_{\odot}$, but notice that this a rather strong function of the assumed outer radius of the CND, because it enters quadratically in the mass density. The number of individual clouds in the CND is only 80 in the FG model.

\subsubsection{Why supernovae do not play any role in the Circumnuclear Disk?}

In a first attempt we followed Paper II and assumed $\delta=5$, but the resulting driving length scale was much to large. We could reduce that problem by choosing $\delta=2$. The relative thickness of the disk is known $\chi=H / R=0.14$, so that we derived from Eq. (14) a mass accretion rate of $\dot{M}=2.3 \times 10^{-3} M_{\odot} \mathrm{yr}^{-1}$. The expression for the volume filling factor (Eq. (16)), the driving length scale (Eq. (15)), and the cloud radius then give $\phi_{\mathrm{V}}=$ $8.2 \times 10^{-4}, l_{\text {driv }}=1.24 \mathrm{pc}$, and $r_{\mathrm{cl}}=l_{\text {driv }} / \delta=0.62 \mathrm{pc}$. In this scenario the cloud radius is derived from the model and is listed in Table 3 for that reason. Consequently, the mass of a single gas cloud is $M_{\mathrm{cl}}=1.6 \times 10^{5} M_{\odot}$ even larger than the whole mass of the CND.

In a further approach we treated $\delta$ as a free parameter, solving Eqs. (15) and (14) together with $r_{\mathrm{cl}}=l_{\text {driv }} / \delta$ leads to the incredible ratio

$\delta=0.86 Q \zeta^{-5} \frac{R}{r_{\mathrm{cl}}} \frac{v_{\mathrm{turb}}^{15}}{v_{\mathrm{rot}}^{11} \xi^{2}}=7.4 \times 10^{6}$,

which states that $l_{\text {driv }} \approx 370 \mathrm{kpc}$.

In a last attempt we left $\xi$ the normalization of the $\mathrm{SN}$ energy input rate as a free parameter. This requires us to fix the driving length scale $l_{\text {driv }}$, which up to now was always too large, to the disk scale height $l_{\text {driv }}=H=1 \mathrm{pc}$. The cloud radius from Table 1 is used as input here and is included in Table 3 for completeness only. This immediately leads to $\delta=20$, an accretion rate of $\dot{M}=6 \times 10^{-3} M_{\odot} \mathrm{yr}^{-1}$, a volume filling factor of $\phi_{\mathrm{V}}=$ $10^{-6}$, and a cloud mass of $M_{\mathrm{cl}}=5 \times 10^{4} M_{\odot}$. Apart from the much too large cloud mass one sees that this model also fails for the SN efficiency, because we find $\xi=2.7 \times 10^{7}(\mathrm{pc} / \mathrm{yr})^{2}$, which requires star formation and the resulting $\mathrm{SN}$ energy input into the disk to be about 600 times more effective than elsewhere in the Galaxy. The large driving length scale or the large star
Table 2. Assumed properties of an obscuring torus.

\begin{tabular}{lll}
\hline \hline sound speed within the clouds & $c_{\mathrm{s}}$ & $1.2 \mathrm{~km} \mathrm{~s}^{-1}$ \\
turbulent velocity dispersion in the disk & $v_{\text {turb }}$ & $72 \mathrm{~km} \mathrm{~s}^{-1}$ \\
disk radius & $R$ & $10 \mathrm{pc}$ \\
disk height & $H$ & $6 \mathrm{pc}$ \\
rotation velocity & $v_{\text {rot }}$ & $120 \mathrm{~km} \mathrm{~s}^{-1}$ \\
\hline
\end{tabular}

formation/SN efficiency required shows, that energy supplied by $\mathrm{SN}$ under normal conditions is much too small to drive the dynamics of the CND.

\subsubsection{A transparent collisional CND}

The observed properties of the CND allow us to estimate the "transparency" $\tau=l_{\text {coll }} / H$ of the CND from Eq. (25) which results in $\tau=15$. So on average there are about $\tau /(2 \pi) \approx 2$ collisions for each cloud per orbit in this model. The expression for the velocity dispersion Eq. (9), which is equally valid for the collisional case with $\zeta=\tau$, gives an estimate of the mass accretion rate of $\dot{M}=1.3 \times 10^{-3} M_{\odot} \mathrm{yr}^{-1}$, which is almost the same as in the FG model, because $\tau$ here and $\zeta$ for in the FG case are not much different. The volume filling factor of clouds $\phi_{\mathrm{V}}=\left(4 r_{\mathrm{cl}} \Omega\right) /\left(3 \tau v_{\text {turb }}\right)=3.6 \times 10^{-3}$ is significantly larger than in the FG model. The reason is that $Q$ enters in $\phi_{\mathrm{V}}$ of the FG model (Eq. (11)), while it is the cloud size in the collisional case here. The result is a substantially smaller mass of typical clouds $M_{\mathrm{cl}}=19 M_{\odot}$. While the transparency derived from Eq. (25) depends on global properties like the total gas mass in the CND, $\tau$ can also be determined from a purely geometrical consideration

$\tau=\frac{\Omega}{n \sigma v_{\text {turb }}}=20$

with the cloud number density $n=N_{\mathrm{cl}} / V=\left(M_{\mathrm{gas}} / M_{\mathrm{cl}}\right) / V$, the total number of clouds $N_{\mathrm{cl}}=1044$, and $V=200 \mathrm{pc}^{3}$ the volume of the disk. This value is reasonably close to the one derived from Eq. (25) above.

\subsection{Building geometrically and optically thick tori}

For obscuring the BLRs in type 2 AGN and for collimating outflows and the ionizing flux in the extended NLR (ionization cones) we suggest properties for an obscuring torus as summarized in Table 2. The values would be appropriate for our galactic center becoming a Sy 2 nucleus with a compact dusty torus. The results are easily rescaled to objects like NGC 1068, where NIR-speckel observations (Wittkowski et al. 1998) show that the inner rim of the torus, if interpreted as such, is less than $2 \mathrm{pc}$ from the AGN. The torus rotates somewhat faster and since there is an enhanced radiation from the active nucleus, the sound speed within the clouds is somewhat higher.

As already mentioned in Sect. 1 the torus is obscuring if the mean free path of the clouds $l_{\text {coll }}$ is smaller than the line of sight through the torus. In a first approximation this corresponds to the condition $l_{\text {coll }}=H$ where we expect that in the midplane 
the line of sight is blocked by several clouds (Nenkova et al. 2002) and the torus is also geometrically thick $H \sim R$. Within the framework of the FG and collisional models this leads immediately to the requirement $\zeta=\tau=1$.

We can start the discussion with the statement that the FG model fails to describe a thick torus, because the requirement $\zeta=1$ implies that the cloud radius equals the disk height $r_{\mathrm{cl}}=H / \zeta=H$. Since this is unphysical, we can reject this model for obscuring tori.

\subsubsection{Colliding clouds in a thick torus}

From the condition that the gas clouds have to resist tidal shear Eq. (23) reads $Q \leq 54$. As the torus is thicker for larger $Q$ we assume $Q$ to take its maximal allowed value. This means that typical clouds are actually at the shear limit. Together with $\tau=1$ we find clouds of $r_{\mathrm{cl}}=0.1 \mathrm{pc}$. From Eq. (9) like in the CND case we find an accretion rate of $\dot{M}=3.3 M_{\odot} \mathrm{yr}^{-1}$ and from Eq. (12) a gas mass in the torus of $M_{\text {gas }}=4 \times 10^{5} M_{\odot}$. The volume filling factor is determined in analogy to the CND case (Sect. 4.1.3) and gives $\phi_{\mathrm{V}}=2.5 \times 10^{-2}$. The resulting mass of a single gas cloud is here $M_{\mathrm{cl}}=45 M_{\odot}$. The total gas mass is larger than in the collisional CND case as expected, because more mass in clouds is needed for obscuration. The implied cloud sizes and cloud masses are surprisingly similar to those of the CND, which may indicate that the dominating physical processes in CND and torus are the same. The volume filling factor is of course larger as we would not get an obscuring torus otherwise. The higher density of clouds now lead to more cloud collisions and consequently the increased mass accretion rate, which feeds the AGN.

\subsubsection{Supernovae can drive tori at large mass accretion rates}

For the SN driven turbulent case of a torus model it is not obvious from the beginning what the appropriate $Q$-parameter is. The torus will only be geometrically thick for large turbulent velocities. This together with the normalization of the SN efficiency $\xi$ derived from integrated galactic values (Paper II), implies that a mass accretion rate (Eq. (13)) of $\dot{M} \sim 45 \delta^{1 / 3} M_{\odot} / \mathrm{yr}$ would be needed for a $(Q=1)$-torus.

In the case of a torus with $Q>1$ we can estimate, if the energy supplied by supernovae will dominate the heating rate due to viscosity and differential rotation. Inserting the volume filling factor from Eq. (16) into Eq. (17) leads to a limit for the product $\delta Q^{2}$ :

$\delta Q^{2} \leq \frac{\zeta^{3} \xi}{\chi^{2} v_{\text {rot }}^{2}}$

With the values for the torus model from Table 2 it gives a limit $\delta Q^{2}<8.6 \zeta^{3}$. The comparison in the inequality (29) is made with the collisional case and for a torus it implies $\zeta=$ $\tau=1$. This results in a severe limit for both $\delta$ and $Q$, because both parameters should be larger than 1 . The range of allowed values for $\delta$ and $Q$ is rather small and we can assume $\delta=2$ and $Q=2$ for the moment. The model is now completely fixed
Table 3. Derived parameters and properties of the CND and an obscuring torus (TORUS). The values in brackets for the $\mathrm{SN}$ model are for $\xi$ treated as a free parameter as described in Sect. 4.1.2.

\begin{tabular}{|c|c|c|c|c|}
\hline & & CND & TORUS & units \\
\hline \multicolumn{5}{|l|}{ FG model } \\
\hline Toomre parameter & $Q$ & 190 & - & \\
\hline$\overline{l_{\text {driv }} / l_{\text {diss }}}$ & $\zeta$ & 20 & 1 & \\
\hline mass accretion rate & $\dot{M}$ & $1 \times 10^{-3}$ & - & $M_{\odot} \mathrm{yr}^{-1}$ \\
\hline volume filling factor $\varphi$ & $\phi_{\mathrm{V}}$ & $2.6 \times 10^{-4}$ & - & \\
\hline cloud mass & $M_{\mathrm{cl}}$ & 260 & - & $M_{\odot}$ \\
\hline \multicolumn{5}{|l|}{ SN model } \\
\hline Toomre parameter & $Q$ & 190 & $\sim 2$ & \\
\hline$l_{\text {driv }} / l_{\mathrm{cl}}$ & $\delta$ & $2(20)$ & $\sim 2$ & \\
\hline mass accretion rate & $\dot{M}$ & $2.3 \times 10^{-3}\left(6 \times 10^{-3}\right)$ & 42 & $M_{\odot} \mathrm{yr}^{-1}$ \\
\hline volume filling factor $\phi$ & $\phi_{\mathrm{V}}$ & $8 \times 10^{-4}\left(10^{-6}\right)$ & $\sim 6 \times 10^{-2}$ & \\
\hline cloud radius & $r_{\mathrm{cl}}$ & $0.62(0.05)$ & 2.0 & $\mathrm{pc}$ \\
\hline cloud mass & $M_{\mathrm{cl}}$ & $1.6 \times 10^{5}\left(5 \times 10^{4}\right)$ & 280 & $M_{\odot}$ \\
\hline \multicolumn{5}{|l|}{ collisional model } \\
\hline Toomre parameter & $Q$ & 190 & $\sim 54$ & \\
\hline$t_{\mathrm{coll}} \Omega$ & $\tau$ & 15 & 1 & \\
\hline mass accretion rate & $\dot{M}$ & $1.3 \times 10^{-3}$ & 3.3 & $M_{\odot} \mathrm{yr}^{-1}$ \\
\hline volume filling factor $\varphi$ & $\phi_{\mathrm{V}}$ & $3.6 \times 10^{-3}$ & $2.5 \times 10^{-2}$ & \\
\hline cloud mass & $M_{\mathrm{cl}}$ & 20 & 45 & $M_{\odot}$ \\
\hline disk mass & $M_{\mathrm{gas}}$ & $2 \times 10^{4}$ & $4 \times 10^{5}$ & $M_{\odot}$ \\
\hline
\end{tabular}

and we find a huge mass accretion rate of $\dot{M}=42 M_{\odot} \mathrm{yr}^{-1}$ through the torus, with cloud sizes of $r_{\mathrm{cl}} \sim 2 \mathrm{pc}$ and cloud masses of $280 M_{\odot}$. The torus in this model has a volume filling factor of clouds of $\phi_{\mathrm{V}} \sim 6 \times 10^{-2}$ and a total mass in gas of $M_{\text {gas }} \sim 1 \times 10^{7} M_{\odot}$. The large cloud sizes and $\delta=2$ implies that the driving length scale is $l_{\text {driv }}=0.7 \mathrm{H}$ almost as large as the torus scale height. Because $Q$ is small in this model and the torus is required to be geometrically thick with $H \sim R$, the gas mass in the torus is comparable to the total enclosed mass in stars and central black hole.

\section{Prerequisites and consequences}

In the previous section we have quantified what is needed for turbulent (fully self-gravitational, or supernovae driven) models and a model consisting of quasi-stable clouds undergoing collisions, which lead to energy dissipation balanced by accretion in the gravitational potential of the galactic center. A résumé of the results for the Circumnuclear Disk and an obscuring torus is given in Table 3.

For the Circumnuclear Disk (CND) in the Galactic Center the model of supernovae driven turbulence ( $\mathrm{SN}$ model) can clearly be rejected, because the inferred cloud mass is larger than $10^{4} M_{\odot}$ and all the mass in the CND would be only in one cloud. Within the framework of the fully gravitational (FG) model, the volume filling factor is small $\left(\phi_{\mathrm{V}}=2.6 \times 10^{-4}\right)$ 
and the cloud mass is about 8 times the observed value $\left(M_{\mathrm{cl}}^{\mathrm{obs}} \sim\right.$ $\left.30 M_{\odot}\right)$. Even when allowing for the uncertainties in the observational parameters like total mass and outer radius of the CND and the crudeness of the approximations in the model, it is nonetheless unlikely that this model is a realistic description of the CND. In contrast to the SN/FG-models the collisional model leads to disk and cloud properties that are close to the observed values. This model thus gives the best approximation to the observations. It shares with the FG model the property that accretion is the energy source for keeping the velocity dispersion, but it consists of stable, individual clouds, which are not part of a turbulent cascade. This conclusion is in qualitative agreement with those already drawn by Krolik \& Begelman (1988). These authors derived the cloud properties on the basis of an extrapolated, observed gas pressure. They then used a model for an equilibrium configuration where cloud mergers balance tidal shearing to show that the cloud area filling factor should be one. In this case the collisional time scale and the time scale for destruction by tidal shear are comparable. In our collisional model, the cloud properties are best described by the transparency $\tau$, which is not fixed to a singular value, and the fact that the clouds must resist tidal shear (Sect. 4.2).

\subsection{The efficiency of star formation and SN energy input in a collisional scenario}

In the following we compare the energy input due to SNe to the energy dissipation rate per unit area due to collisions within the collisional model described above. Supernovae can be important if their energy supplied to the ISM dominates the dissipation rate due to collisions. This is nessesary but not sufficient to influence the dynamics of clouds, as we show below based on the ratio of cloud to $\mathrm{SN}$ remnant (SNR) surface area.

For the first step of the argument the energy supply rate from Eq. (4), modified by an efficiency $\eta$, must be larger than the dissipation rate due to collisions. The star formation rate in a collisional scenario can be estimated following the discussion of Eq. (19) in Paper II by $\dot{\Sigma}_{\star}=\phi_{\mathrm{V}} \Sigma / t_{\mathrm{ff}}$. The free-fall timescale is relevant for the size of clouds in the collisional case (Eq. (20)) and $r_{\mathrm{cl}}$ taken from Eq. (21) with $Q$ at its maximum (Eq. (23)) leads to the relation

$\eta \xi \dot{\Sigma}_{\star}=\frac{\sqrt{8}}{\pi} \eta \phi_{\mathrm{V}} \xi \Sigma \Omega>\Sigma \frac{v_{\mathrm{turb}}^{3}}{\tau H}$.

From that we get a minimum efficiency $\eta_{\mathrm{eq}}$ for which $\mathrm{SNe}$ and accretion supply equal amounts of energy to be dissipated:

$\eta_{\text {eq }}=\frac{\pi}{\sqrt{8}} \frac{v_{\mathrm{turb}}^{2}}{\tau \phi_{\mathrm{v}} \xi}$.

For the collisional torus model described in Sect. 4.2.1 we find $\eta_{\mathrm{eq}}=5.2$ so that star formation and $\mathrm{SNe}$ are required to be more efficient than in galactic disks to become important. If the energy of SNe would be kept in the cloudy structure of the CND the equilibrium value would be $\eta_{\text {eq,CND }}=0.13$, where we have used the "observed" value for $Q$ from Eq. (26). We conclude that if star formation in the Galactic Center proceeds in the same way as in the Galactic disk, SN will provide a perhaps not negligible energy input in addition to dissipative collisions.
Nevertheless, an equilibrium disk scenario where the dynamics of clouds is driven by the energy input due to $\mathrm{SNe}$ is quite unlikely as we will show next. The SNR will expand into the inter-cloud medium (ICM). The ICM density and thermal velocity will limit the size the SNR and therefore the sphere of influence of the SN. To get an estimate for the ICM density we assume that it is in pressure balance with the clouds and fills the same region of space as the clouds do, which implies that the sound speed in the ICM equals the velocity dispersion $v_{\text {turb }}$. The ICM density is therefore $\rho_{\text {ICM }}=\rho_{\mathrm{Cl}} c_{\mathrm{s}}^{2} / v_{\text {turb }}^{2}$. With the volume filling factor of the collisional model

$\rho_{\mathrm{ICM}}=\frac{6}{\pi^{2}} \frac{\tau^{2}}{Q} \rho$,

where $\rho$ is the mean density in torus or CND. It turns out that the ICM density is $70 \%$ of the mean density for the CND and about $1 \%$ for the torus model; in both cases we find a particle density of roughly $10^{3} \mathrm{~cm}^{-3}$, which is in nice agreement with the result of Erickson et al. (1994). We can now estimate the maximum SNR size by using the power law fits provided by Thornton et al. (1998). To do that one has to realize that the SNR resolves when the shock speed of the SNR falls to the sound speed in the external medium which is the velocity dispersion $v_{\text {turb }}$ here. With $v_{\mathrm{s}} \propto R_{\mathrm{SN}}^{-2}$ we get from extrapolating the Thornton et al. result

$R_{\mathrm{SN}}=1.1 E_{51}^{2 / 7} n_{3}^{-0.42}\left(Z / Z_{\odot}\right)^{-0.1} \mathrm{pc}$.

In the CND most SNR are likely to break out of the relatively thin disk of molecular clouds and the efficiency will be largely reduced by this effect. In the case of the torus the fraction of SN energy impinging on clouds is simply the cross section of one cloud times the mean number of clouds within the SNR of radius Eq. (33) divided by the surface area of the SNR. This results in $\eta=R_{\mathrm{SN}} \phi_{\mathrm{V}}^{2} /\left(4 r_{\mathrm{cl}}\right)=\phi_{\mathrm{V}} R_{\mathrm{SN}} /(3 \tau H)$ and the efficiency of $\mathrm{SNe}$ energy from star formation to drive cloud motion is $\eta=1.5 \times 10^{-3}$ for the torus model. Thus we conclude that both CND and obscuring torus can be successfully described by the collisional model. The SN energy will almost exclusively heat the inter-cloud medium and they will have little effect on the dynamics of clouds in a scenario where clouds are stable and "long-lived". Krolik \& Begelman (1988) used different arguments to estimate the energy input due to SNe and stellar winds, which is necessary to maintain a thick torus. They also found that the stirring of the circumnuclear material due to stellar driven processes (supernovae and/or stellar winds) is always weaker than the effects of cloud collisions.

\subsection{The transition from a CND to an obscuring torus and vice versa}

From the discussion up to now it seems that both, the Circumnuclear Disk in the Galactic Center and an obscuring torus, are well described by our collisional model. The density of clouds within the model relative to the critical density against tidal shear $\rho_{\text {crit }}$ is $\rho_{\mathrm{cl}} / \rho_{\text {crit }}=4 /\left(3 Q \phi_{\mathrm{V}}\right)$. For the CND we find $\rho_{\mathrm{cl}} / \rho_{\text {crit }}=1.8$ and the model for the obscuring torus is constructed in a way so that $Q$ takes its critical value from 
Eq. (23) which automatically means that clouds are marginally stable against tidal shear. Both clump densities are close to the critical density with respect to tidal shear and we may speculate that both structures are governed by the same physical processes.

In this sense the CND would be an obscuring torus if it had more mass and it would be thick if it had a higher velocity dispersion. We can quantify this with the definition of the (integrated) Toomre parameter $Q$ (Eq. (2)) to get $\frac{H}{R}=Q \frac{M_{\mathrm{gas}}}{M_{\mathrm{tot}}}$, where $M_{\text {tot }}$ is the total enclosed mass and $M_{\text {gas }}$ the enclosed gas mass at that radius. If clouds are indeed at the shear limit (Eq. (23)) we can replace $Q$ and get an expression for the transparency

$$
\tau=\frac{\pi}{\sqrt{8}} \frac{c_{\mathrm{s}}}{v_{\mathrm{rot}}} \frac{M_{\mathrm{tot}}}{M_{\mathrm{gas}}}
$$

Because total mass and rotation velocity are given by the environment in the galactic center and the sound speed within the clouds is almost constant, we see that the torus becomes obscuring (that means $\tau \sim 1$ ) for large gas masses in the torus. Inserting Eq. (34) and the corresponding expression for $Q$ in Eq. (9) to derive the required accretion rate shows that this rate is $\dot{M} \propto(H / R) v_{\text {turb }} M_{\text {gas }}^{2}$. Therefore large gas masses in a geometrically thick torus can easily provide the accretion rate needed to trigger and maintain nuclear activity. The Eq. (34) for the transparency also shows that the torus around an AGN, which runs out of fuel and has less mass in its surrounding cloud distribution, will become transparent and eventually turn into a CND. Activity in a galactic center including a possibly obscuring torus can also be restarted when additional gas masses in the form of molecular clouds arrive in the central region.

With the critical gas mass for obscuration $(\tau=1)$ from Eq. (34) we can further see that the required mass accretion rate through the torus is

$$
\dot{M}=\frac{\pi}{\sqrt{2}}\left(\frac{H}{R}\right)^{2} c_{\mathrm{s}} \frac{M_{\mathrm{tot}}}{R} .
$$

If the inner radius is set by the dust sublimation radius (see below) and the temperature in clouds is determined by radiative equilibrium with the central source, the sound speed is constant at the inner radius independent of the accretion rate or luminosity. With $\left(R=R_{\text {in }} \propto \dot{M}^{1 / 2}\right)$ we find that the minimum accretion rate for obscuration is

$\dot{M}_{\mathrm{obs}}=3.1 \frac{M_{\odot}}{\mathrm{yr}} \eta_{-1}^{-1 / 3} M_{7}^{2 / 3}\left(\frac{H}{R}\right)^{4 / 3}\left[\frac{c_{\mathrm{s}}}{1.5 \mathrm{~km} \mathrm{~s}^{-1}}\right]^{2 / 3}$,

where $\eta_{-1}$ is the radiation efficiency at the black hole relative to $10 \%$ efficiency and $M_{7}$ the enclosed mass in units $10^{7} M_{\odot}$. In addition it is assumed that all the mass transported through the torus actually reaches the black hole. The required mass accretion rates are highly super-Eddington $\dot{M}_{\text {Edd }}=$ $0.22 M_{\mathrm{BH}, 7} M_{\odot} / \mathrm{yr}$ for the central black hole with $10 \%$ efficiency for transforming rest mass energy into radiation. The solution is that most of the mass accreted through the torus is not effectively turned into radiation at the black hole. It is either blown away in an outflow or jet before reaching the black hole, and thus constitutes a cooling process for the remaining matter, or the accretion process is indeed radiatively inefficient at these super-Eddington rates.

Since these collisional disks are stable and long-lived, a possible formation mechanism of a thick, obscuring torus might be the infall of large gas clumps onto a pre-existing circumnuclear ring as investigated by Vollmer \& Duschl (2002). Infalling gas clouds with different angular momentum will also lead to increased velocity dispersion in the merged system as required for a torus.

\subsection{The physics of clouds and the inner boundary of a torus}

In Vollmer \& Duschl (2001a,b) we investigated the physics of gas clouds in a turbulent or collisional, clumpy disk. It turned out that under realistic conditions, where the gas is heated by the UV radiation of the central HeI star cluster, the gas clouds are stable and self-gravitating. Their radius is mainly determined by the location of the ionization front due to the same UV radiation. Under realistic condition this results in the observed cloud size of $\sim 0.1 \mathrm{pc}$. The present study suggests that the gas and dust physics of thick, obscuring tori is of similar nature.

In this picture two selection effects operate on the initial cloud mass distribution: (i) only clumps that are dense enough to resist tidal shear can survive and (ii) clumps that are more massive than the Jeans mass collapse. In addition, the central UV radiation field determines the size of the clumps, i.e. their mass for a given central density. For the CND it turned out that at a critical distance of $\sim 1.5 \mathrm{pc}$ from the Galactic Center the clouds, which are dense enough to resist tidal shear, will collapse. This is in agreement with the observed inner edge of the CND. If such a mechanism is also at work in a thick, obscuring torus around an AGN, the inner edge of this torus would be determined in the same way, if the dust sublimation radius and the cloud evaporation radius are smaller than the critical radius for collapse and shear (C\&S-radius) described here.

Dust sublimation due to the radiation of a central source determines the size of the inner dust free cavity, which will set the source size for NIR continuum observations (e.g. Wittkowski et al. 1998 for NGC 1068). This is also the inner radius where cool clouds are shielded by dust opacity. A possible description of the sublimation radius by Dopita et al. (1998)

$R_{\text {sub }}=0.84 \mathrm{pc}\left[\frac{T_{\text {sub }}}{1200 \mathrm{~K}}\right]^{-2.27} L_{44}^{1 / 2} a_{-5}^{-1 / 2}$,

shows that $R_{\text {sub }}$ is a strong function of the sublimation temperature $T_{\text {sub }}$, which depends on the dust chemistry and grain sizes with $a_{-5}$ the typical grain radius in units of $10^{-5} \mathrm{~cm}$. The determination of the sublimation radius assumes radiative equilibrium in the dust sublimation region and is due to the $R^{-2}$-dilution of the flux from the central luminosity $L_{44}$ in units of $10^{44} \mathrm{erg} / \mathrm{s}$.

If the dust sublimation radius is larger than the C\&Sradius described above, clouds with masses larger than $\sim 10 M_{\odot}$ can only survive up to this radius. Inside, only the stripped cores of such clouds can survive (the "light clouds" in 
Vollmer \& Duschl 2001a), because the location of the ionization front is determined by self-shielding and not dust absorption.

If the cloud evaporation radius is larger than the C\&S-radius, clouds evaporate before they can reach the C\&S-radius or dust sublimation radius. Following Krolik \& Begelman (1988) clouds will evaporate when the evaporation time scale equals the accretion time scale at a given distance from the ionizing source, which leads to at a typical evaporation radius of

$\frac{R_{\text {evap }}}{\mathrm{pc}}=\frac{0.79 M_{\mathrm{gas}, 5} L_{44}}{\dot{M}_{\mathrm{torus}} R_{\text {out }} N_{\mathrm{cl}, 24} T_{5}^{1 / 2}}$,

for a cloud with a gas column density in units of $10^{24} \mathrm{~cm}^{-2}$, a temperature at the sonic point of the evaporating flow $T_{5}$ of $10^{5} \mathrm{~K}$, a central ionizing luminosity $L_{44}$ in units of $10^{44} \mathrm{erg} \mathrm{s}^{-1}$, the mass accretion rate $\dot{M}_{\text {torus }}$ measured in $M_{\odot} / \mathrm{yr}$, the total gas mass in clouds $M_{\text {gas }, 5}$ relative to $10^{5} M_{\odot}$, and the outer radius of the torus $R_{\text {out }}$ in parsec. Here we assumed $\Sigma \propto R^{-1}$, which is expected for a constant rotation curve (the torus residing in the potential of an isothermal star cluster). Inserting the values for the collisional torus model from Table 3 one finds $R_{\text {evap }}=0.1 \mathrm{pc}$. The evaporation radius is sensitive to the ionizing luminosity and $N_{\mathrm{cl}}$ in an individual cloud, so that Eq. (38) can be used to set a lower limit to the column density at a given radius. Equation (38) seriously underestimates the evaporation radius if it gives $R_{\text {evap }}>R_{\text {out }} / 2$. We can use this in the collisional model discussed here for a central source radiating at the Eddington limit of a $3 \times 10^{6} M_{\odot}$ black hole and find that only clouds with $N_{\mathrm{cl}}>2 \times 10^{22} \mathrm{~cm}^{-2}$ will survive when they are exposed to the central radiation source. The clouds in the model with $r_{\mathrm{cl}}=0.1 \mathrm{pc}$ and $45 M_{\odot}$ are safely above that limit. At an inner edge of about $2 \mathrm{pc}$ the clouds have a column density of $\geq 10^{24} \mathrm{~cm}^{-2}$ (assuming $\phi_{\mathrm{V}}$ a constant) and all clouds in the model are stable against evaporation.

\section{Conclusions}

We explored three distinct models of clumpy gas disks for the Circumnuclear Disk (CND) in the Galactic Center and for a geometrically thick, obscuring torus, as suggested in unified models of AGN. Three kinds of models were tested: (i) a turbulent, fully gravitational model, where the energy, which is gained by mass inflow and differential rotation, is cascaded by turbulence to smaller scales. Alternatively (ii) the turbulence may be maintained by the energy input due to supernova explosions, and we investigated (iii) a collisional model, where the gas viscosity is due to partly dissipative cloud collisions. In the cases (i) and (ii) we identify the energy sink to be the self-gravitation of the gas clouds, which separates them from the turbulent cascade.

We find that the fully gravitational model cannot be entirely excluded for the case of a CND. The cloud masses for the
CND model are larger than indicated by observations, but the masses in the model depend strongly on the assumed outer radius for an equilibrium disk structure. A turbulent cascade may also exist in a massive torus at exceedingly large mass accretion rates, where supernovae can drive the turbulence. Nonetheless the CND and a thick, obscuring torus are best described by the collisional disk model. We suggest that both the CND and a thick, obscuring torus share the same gas physics: they consist of stable, self-gravitating clouds that are heated by the central UV radiation and the density of clouds is in both cases close to the critical density with respect to tidal shear. The mass of single clouds is a few times $10 M_{\odot}$ in the CND and in a torus.

In this picture a thick, obscuring torus is a more massive CND-like structure, that is with more gas clumps, and in addition a higher velocity dispersion. We show that a torus provides automatically the high mass accretion rate needed to trigger and maintain nuclear activity and that a geometrically thick torus is obscuring the view to the center only at super-Eddington rates.

The model for clumpy collisional disks suggests that the appearance as a CND or an obscuring torus only depends on the mass supply from larger radii in the galaxy, possibly in the form of pieces of giant molecular clouds.

Acknowledgements. Part of this work was support by the DFG through grant SFB439 (WJD).

\section{References}

Antonucci, R. 1993, ARA\&A, 31, 473

Dopita, M. A., Heisler, C., Lumsden, S., \& Bailey, J. 1998, ApJ, 498, 570

Erickson, E. F., Colgan, S. W. J., Simpson, J. P., Rubin, R. H., \& Hass, M. R. 1994, Far-Infrared Line Observations of Sgr A West. In The Nuclei of Normal Galaxies, ed. R. Genzel, \& A. I. Harris, NATO Advanced Science Institutes Series C, 445, 249

Güsten, R., Genzel, R., Wright, M. C. H., et al. 1987, ApJ, 318, 124

Jackson, J. M., Geis, N., Genzel, R., et al. 1993, ApJ, 402, 173

Krolik, J. H., \& Begelman, M. C. 1988, ApJ, 329, 702

Mulchaey, J. S., Wilson, A. S., \& Tsvetanov, Z. 1996, ApJ, 467, 197

Nenkova, M., Ivezic, Z., \& Elitzur, M. 2002, ApJ, 570, L9

Pringle, J. E. 1981, ARA\&A, 19, 137

Schmitt, H. R., Antonucci, R. R. J., Ulvestad, J. S., et al. 2001, ApJ, 555,663

Schödel, R., Ott, T., Genzel, R., et al. 2002, Nature, 419, 694

Tran, H. D. 2003, ApJ, 554, L19

Thornton, K., Gaudlitz, M., Janka, H.-Th., \& Steinmetz, M. 1998, ApJ, 500, 95

Vollmer, B., \& Duschl, W. J. 2001, A\&A, 367, 72

Vollmer, B., \& Duschl, W. J. 2001, A\&A, 377, 1016

Vollmer, B., \& Duschl, W. J. 2002, A\&A, 388, 128

Vollmer, B., \& Beckert, T. 2002, A\&A, 382, 872 (Paper I)

Vollmer, B., \& Beckert, T. 2003, A\&A, 404, 21 (Paper II)

Wilson, A. S. 1996, VA, 40, 63

Wittkowski, M., Balega, Y., Beckert, T., et al. 1998, A\&A, 329, L45-L48 